\section{UPS AND DOWNS}

The numbers of human cases of H7N9 avian flu diagnosed plummeted last year after live-bird markets were closed, but have since resurged.

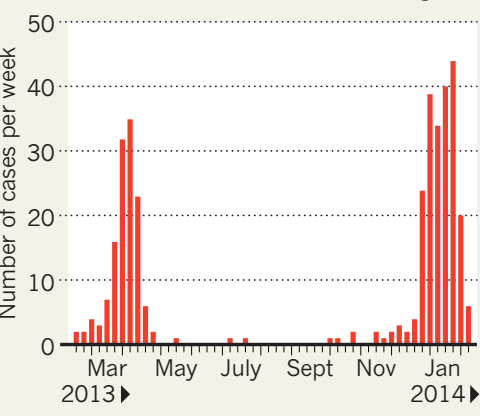

of Guangzhou in Guangdong province began closing its markets on that day too.

"Interventions were clearly less stringent this year," says Ben Cowling, a flu epidemiologist at the University of Hong Kong. "My impression is that financial concerns had a greater influence this winter compared to last spring." The Chinese poultry industry has vigorously protested against the policy of shutting down markets.

But the surge in H7N9 flu cases highlights the continuing public-health and possibly pandemic - threat that it poses. The virus's epidemiology remains largely unchanged from last year: it is still, essentially, an avian virus that is sporadically infecting humans from a reservoir in poultry, and there is no evidence of any continued human-to-human spread. But the virus is being sustained in unknown reservoirs in the poultry supply chain, making future outbreaks likely, and its geographical expansion is a reminder of the threat beyond China. Furthermore, although case numbers have shown signs of dropping in the past two weeks, it is too early to tell if this marks a clear trend.

One year on from the first outbreak, researchers are still struggling to understand the origins and dynamics of the virus's reservoirs and spread. H7N9 surveillance is difficult, in part because the virus causes only mild disease in poultry and thus spreads silently, with human cases typically the first warning of a poultry outbreak. $\mathrm{H} 5 \mathrm{~N} 1$, by contrast, is lethal to birds, making outbreaks easier to spot and control. And despite extensive sampling of farms, wholesale markets and other parts of the poultry supply chain, the only strong link to H7N9 found so far is live-bird markets.

The difficulty of surveillance and of sampling China's huge poultry industry - it produces 6 billion birds annually means that this is unlikely to be the full picture, however. Such uncertainty is also hampering efforts to develop effective control measures that are less drastic than closing the markets, says Cowling.

NEUROSCIENCE

\title{
Monkey brains wired to share
}

\section{Game-theory test exposes circuits for social interaction.}

\section{BY SARA REARDON}

$\mathrm{T}$ Iwo monkeys sit at computer screens, eyeing one another as they wait for a promised reward: apple juice. Each has a choice - it can either select a symbol that results in juice being shared equally, or pick one that delivers most of the juice to itself. But being selfish is risky. If its partner also chooses not to share, neither gets much juice.

This game, the 'prisoner's dilemma', is a classic test of strategy that involves the simultaneous evaluation of an opponent's thinking. Researchers have now discovered - and manipulated - specific brain circuits in rhesus macaques (Macaca mulatta) that seem to be involved in the animals' choices, and in their assessments of their partners' choices. Investigating the connections could shed light on how social context affects decision-making in humans, and how disorders that affect social skills, such as autism spectrum disorder, disrupt brain circuitry.

"Once we have identified that there are particular neural signals necessary to drive the processes, we can begin to tinker," says Michael Platt, a neurobiologist at Duke University in Durham, North Carolina.

Neurobiologists Keren Haroush and Ziv Williams of Harvard Medical School in Boston, Massachusetts, zoomed in on neural circuits in rhesus macaques by implanting electrode arrays into a brain area called the dorsal anterior cingulate cortex (dACC), which is associated with rewards and decision-making. The arrays recorded the activity of hundreds of individual neurons. When the monkeys played the prisoner's dilemma (see 'A juicy experiment') against a computer program, they rarely chose to cooperate. But when they played with another monkey that they could see, they were several times more likely to choose to share the juice.

As the monkey made its decision, a specific set of dACC neurons tended to fire if the animal was choosing to cooperate, allowing the researchers to predict its decision two-thirds of the time. A different set often fired if the monkey thought its partner would cooperate, and the predictions were $80 \%$ accurate.

Researchers have long known about mirror neurons, which are involved in copying other individual's actions, but this is the first discovery of neurons that predict another individual's unknown actions. The researchers also found that when they interfered with the cooperation circuit by delivering an electric shock to the area, the monkeys became less likely to cooperate - suggesting that the circuits for social interaction normally override an inherent desire to self-indulge. Haroush and Williams will present their findings this week at the Computational and Systems Neuroscience meeting in Salt Lake City, Utah.

The ability to track individual circuits while monkeys assess one another is an important advance, says Robert Seyfarth, a psychologist at the University of Pennsylvania in Philadelphia, although he cautions that real social interactions are much more complex. Furthermore, he says, a monkey's willingness to cooperate might depend on the other monkey - whether it is a dominant male, for instance, or a relative. "We really need to ask monkeys what they know about other monkeys," says Seyfarth.

A closer look at the circuits could reveal

\section{A JUICY EXPERIMENT}

Researchers have monitored the brains of monkeys playing the prisoner's dilemma strategy game. If both monkeys pick the 'share' symbol, they split an apple-juice reward. If both pick 'hoard', neither gets much juice. If one hoards while the other shares, it gets most of the juice - but its partner may be less likely to share later.

1 Monkeys choose

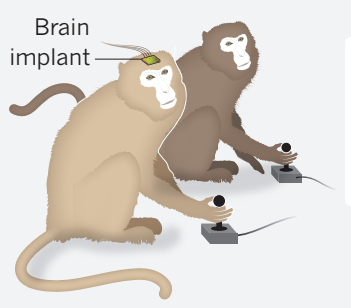

2 Choices revealed

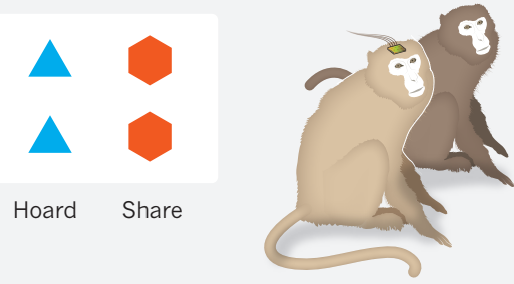


zow they respond to hormones or drugs, says Steve Chang, a psychologist at Yale University in New Haven, Connecticut. The neurohormone oxytocin, in particular, has been touted as a potential treatment for autism, because it seems to aid the formation of social bonds. For example, men playing the prisoner's dilemma are more likely to cooperate if they have breathed in oxytocin ${ }^{1}$.

Chang and Platt have monitored the brains of monkeys playing a different game, in which they can choose to reward other monkeys without any sacrifice. Receiving a reward causes one set of neurons to fire, they found ${ }^{2}$; watching another monkey receive a reward triggers a different set. As in the prisoner's dilemma, the monkeys wanted to reward another monkey only if it was physically present, and were unlikely to reward a computer. Chang is now studying how oxytocin, which has been shown to increase monkeys' willingness to reward ${ }^{3}$, affects neural circuitry.

Besides drugs and neurohormones, electrical stimulation can also alter the brain. Platt's lab is trying to map neural circuits, and show how they respond to electromagnetic stimulation. Previous work has shown ${ }^{4}$ that stimulating certain parts of the brain can increase people's ability to perform empathetic tasks, such as assessing what someone else knows or likes.

But Platt says that scientists are just beginning to understand how such methods work. He adds that watching the response of neural circuits in monkeys is a good way to work out how much stimulation or hormone should be applied, and where. "If you were a parent and considering oxytocin or brain stimulation for your child, you would want to know the answers to all those questions," he says. -

1. Rilling, J. K. et al. Psychoneuroendocrinology 37, 447-461 (2012).

2. Chang, S. W. C., Gariépy, J.-F. \& Platt, M. L. Nature Neurosci. 16, 243-250 (2013).

3. Chang, S. W. C., Barter, J. W., Ebitz, R. B. Watson, K. K. \& Platt, M. L. Proc. Natl Acad. Sci. USA 109, 959-964 (2012).

4. Santiesteban, I., Banissy, M. J., Catmur, C. \& Bird, G. Curr. Biol. 22, 2274-2277 (2012).

3 Rewards delivered

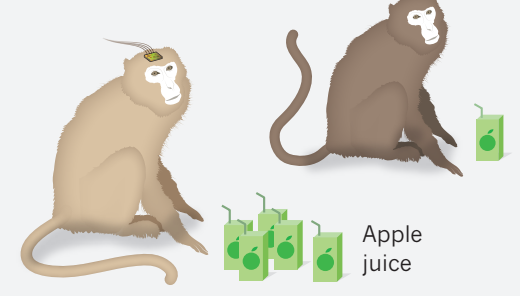

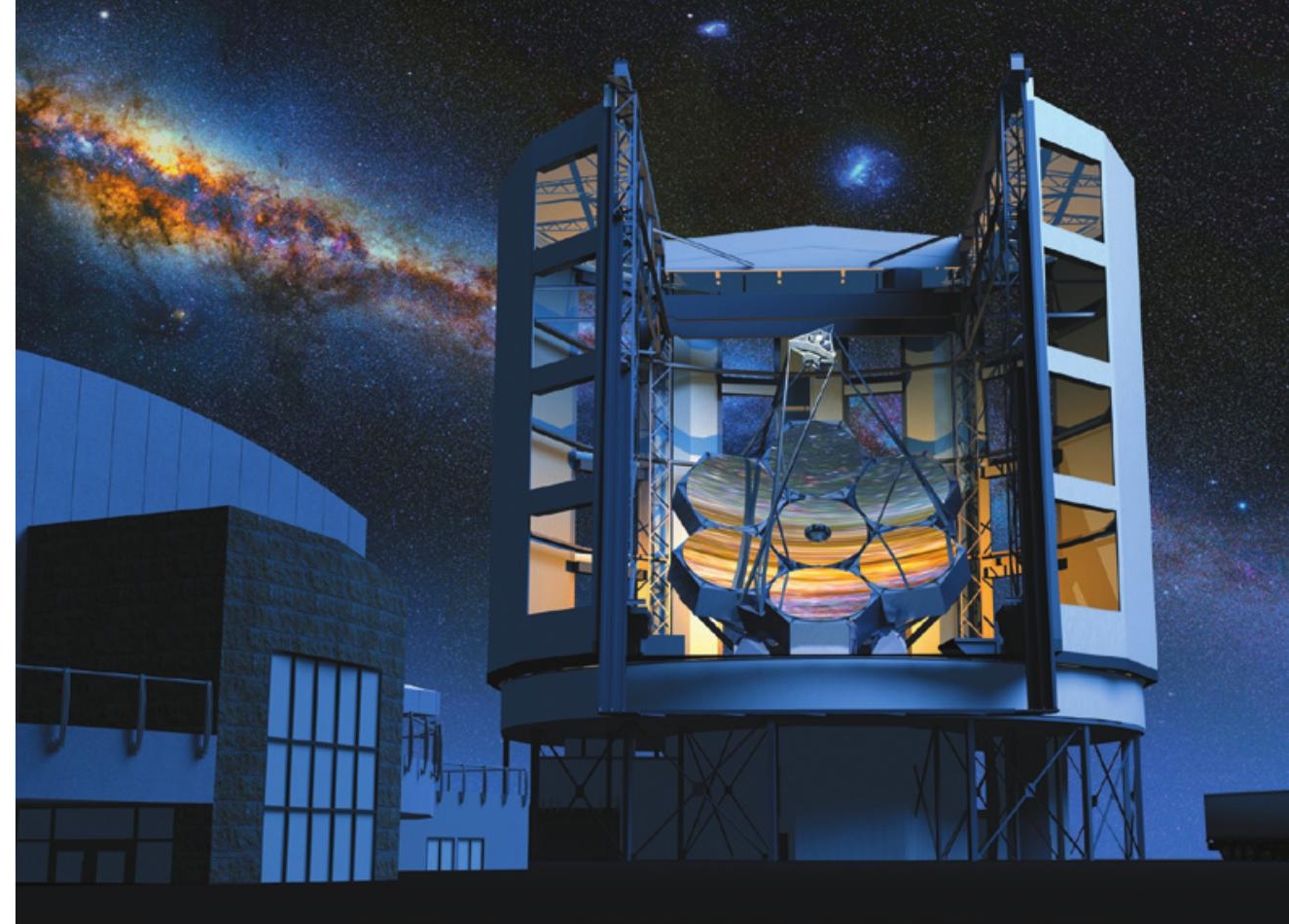

The proposed Giant Magellan Telescope in Chile is seeking to secure funding for its construction phase.

ASTRONOMY

São Paulo poised to
join megatelescope

Brazilian state mulls support for Giant Magellan Telescope.

\section{BY ELIZABETH GIBNEY}

A stronomers in Brazil's richest state are pushing for a stake in the Giant Magellan Telescope (GMT), one of three planned megatelescopes. The São Paulo Research Foundation (FAPESP) is evaluating a proposal that would see it invest US\$40 million in the 25-metre facility, to be built at the Carnegie Institution for Science's Las Campanas Observatory in Chile.

If Brazil finalizes joining the European Southern Observatory (ESO) as planned under a separate proposal, São Paulo state astronomers would have access to another megatelescope, the 39-metre European Extremely Large Telescope (E-ELT), which ESO also plans to build in Chile. Brazil's accession agreement to ESO, signed in 2010 by the then science minister Sergio Machado Rezende, would deliver $€ 270$ million (US\$371 million) to the organization over a decade - almost one-third of the E-ELT's cost. But Brazil's legislative committees are still hunting for funds and its Congress has not ratified the agreement (see Nature 501, 13-14; 2013).

The strength of the astronomy department at the University of São Paulo alone helps to explain the state's move to join the GMT, says Gary Sanders, project manager of the Thirty Meter Telescope, the third megatelescope in the pipeline, planned for Mauna Kea in Hawaii. "As an outsider, it does seem natural for me for the state of São Paulo to provide access to an extremely large telescope for that community," he says.

The GMT project is managed by a consortium of institutions in the United States, Australia and South Korea. The telescope will study the skies with about six times the collecting power of the largest existing observatories, probing deeply into how stars and galaxies formed in the early Universe. On 19 February, the GMT board announced that a major milestone had been cleared when a panel of experts approved its design. Now that the $\$ 880$-million telescope is technically ready for construction, it must secure its financial commitments. Cash is exactly what São Paulo could provide.

The state generates one-third of Brazil's gross domestic product, and more than half of its scientific output. São Paulo produces more scientific articles than any South American country, aside from Brazil as a whole. Funding for the GMT would bypass complicated federal mechanisms and come directly from FAPESP, which is supported by $1 \%$ of state taxes.

The proposal before FAPESP would secure a $4 \%$ stake in the GMT, guaranteeing São Paulo's researchers $4 \%$ of observation time each year, as well as representation on the consortium's decision-making board. This would be a 